9. Yeghiazarians Y, Braunstein JB, Askari A, Stone PH. Unstable angina pectoris. N Engl J Med 2000;342:101-4.

10. Coller BS. Anti-GPIIb/IIIa drugs: current strategies and future directions. Thromb Haemost 2001;86:427-43.

11. Gallus AS, Baker RI, Chong BH, Ockelford PA, Street AM. Consensus guidelines for warfarin therapy. Recommendations from the Australasian Society of Thrombosis and Haemostasis. Med J Aust 2000;172:600-5. http://www.mja.com.au/public/issues/172_12_190600/gallus/gallus.html

12. Makris M, Watson HG. The management of coumarin-induced over-anticoagulation. Br J Haematol 2001;114:271-80.

13. Interaction of celecoxib and warfarin. Aust Adv Drug React Bull 2001;20:1. 14. Interaction of rofecoxib with warfarin. Aust Adv Drug React Bull 2002;21:1.

15. Beyth RJ, Quinn LM, Landefeld CS. Prospective evaluation of an index for predicting the risk of major bleeding in outpatients treated with warfarin. Am J Med 1998;105:91-9.

16. Gage BF, Fihn SD, White RH. Management and dosing of warfarin therapy. Am J Med 2000;109:481-8.
FURTHER READING

Campbell P, Roberts G, Eaton V, Coghlan D, Gallus A. Managing warfarin in the community. Aust Prescr 2001;24:86-9.

\section{Conflict of interest: none declared}

\section{Self-test questions}

The following statements are either true or false (answers on page 99)

1. The INR is not affected by COX-2 inhibitors.

2. Non-steroidal anti-inflammatory drugs have a more prolonged antiplatelet effect than aspirin.

\title{
Managing constipation in children
}

Constipation, defined as difficulty, delay or pain on defecation, is common in children and is often difficult to manage. Here, we review the assessment and treatment of affected children and the support that they, and their families, may need.

\section{Background}

Breastfed babies have a mean of about three bowel movements daily, while formula-fed babies have about two. With age, the frequency falls to about one movement daily in children over three years. ${ }^{1}$ In one study, $96 \%$ of children aged $1-4$ years had somewhere between one bowel movement every other day to three movements daily. ${ }^{2}$ So as long as the child is pain free, parents can usually be reassured that some infrequency in defecation is likely to be normal.

Various factors may cause or increase the likelihood of constipation. Delay in passing meconium more than 48 hours after birth, or constipation in early infancy, suggests the possibility of Hirschsprung's disease, especially if there is also excessive vomiting, abdominal distension or failure to gain weight. Constipation may result from inadequate food or fluid intake, while children who drink a lot of milk may have hard stools that are difficult to pass. Chronic constipation might be associated with an intolerance to cow's milk ${ }^{3}$, although this is more commonly associated with diarrhoea. Some medicines can induce constipation (e.g. opiate analgesics, anticholinergic drugs). Children may withhold defecation and this may make them liable to constipation. Risk factors for withholding include: 'fear' of previous treatments for constipation or coercive potty training, sometimes coinciding with the developmental stage between two and three years of age when children typically refuse to obey their parents; lack of privacy (especially in school lavatories); domestic stress; sexual abuse; or pain on defecation due to anal fissures or a perianal skin infection.

When the rectum is chronically obstructed with faeces (or incompletely emptied), it may enlarge to form a megarectum. Children with a megarectum may not sense the faecal matter in the rectum, and have diminished urgency to defecate ${ }^{4,5}$, which may lead to faecal soiling. It is important to distinguish this involuntary soiling from encopresis, in which the child voluntarily passes normal stools in unacceptable places.

\section{Assessment}

It is important to take a detailed history of the illness from the parent and, where possible, the child, including noting of relevant dietary, family and social factors. Clinical examination should appraise the child's general health and check for poor growth and neurological problems. Palpation of the abdomen may reveal distension or faecal loading in the colon. Rectal examination can be distressing for the child and is usually unnecessary. In most instances, inspection of the perineum is sufficient to check for the presence of anal fissures, infection, skin disease, anal ectopia or anal abuse.

There is no need for a routine abdominal X-ray to diagnose constipation $^{6}$, but it may help confirm overflow incontinence in a child with faecal impaction who initially presents with diarrhoea. A potentially more helpful hospital investigation involves the child swallowing radio-opaque gut transit markers over three days and then taking an abdominal X-ray on day five. ${ }^{7}$ This test may confirm: fast intestinal transit in children with episodes of faecal incontinence; rectal retention in children with megarectum; or pancolonic delay (colonic inertia) in older children.

\section{Treatment}

The general principles in managing childhood constipation are to: clear any faecal impaction; establish a regular and effective pattern of defecation; and to prevent recurrence. ${ }^{1,8}$ Where possible, underlying causes should be resolved, for example, stopping constipating medicines, treating painful anal conditions and addressing possible psychosocial causes. There is a lack of evidence from randomised controlled trials to guide management choices.

\section{Dietary intervention}

Constipation can often be relieved by increasing dietary fluid and fibre. However, children may be reluctant to eat high-fibre foods such as fruit, vegetables and cereals, especially if the rest of the family eat a different diet. If the child's appetite is poor, 
this needs investigation, particularly if food is avoided because of discomfort after eating. However, parents can usually be reassured that faddy eating is common and advised to avoid being too anxious at mealtimes. Rearrangement of mealtimes may help where the child withholds defecation at school; for example, eating breakfast earlier might enable the child to open his or her bowels before leaving home. The general practitioner is well placed to provide general dietary advice and reassurance, but referral to a paediatrician or child psychiatrist may be necessary where major feeding problems exist. Some infants who take large quantities of formula milk may benefit from a reduced intake. Substantial changes or restriction of dietary intake should be supervised by a paediatrician or dietitian with paediatric experience.

\section{Laxative treatments}

If dietary changes are not sufficient to produce softer and more frequent stools, starting a laxative may help. If the child is old enough, it is important to explain to them why laxatives are being given. Treatment should start with regular doses of a stool softener/osmotic laxative (e.g. lactulose) or a bulkforming laxative (e.g. ispaghula husk, methylcellulose) to produce a soft, easily passed stool. If these drugs do not work, or if the child is withholding defecation, a stimulant laxative should be tried (e.g. senna, bisacodyl or sodium picosulfate syrup). These laxatives stimulate colonic propulsion, which quickens the filling of the rectum and intensifies rectal contractions. Defecation is therefore more frequent and so the stool is smaller and softer, which gradually reduces the child's fear of the sensation of imminent defecation. In a crossover study involving 21 children (aged under 15 years) with chronic constipation, lactulose was more likely to lead to passage of normal stools than senna and more unwanted effects (colic, diarrhoea) were noted with senna treatment. ${ }^{9}$ However, a combination of laxatives (e.g. lactulose and senna) may be particularly effective and should be considered if individual drugs fail. If the child has a megarectum impacted with hard stools, stimulant laxatives may aggravate overflow faecal incontinence and abdominal pain; using docusate, a stimulant laxative with stool-softening properties, is a reasonable option in this situation. If this is unsuccessful glycerine suppositories or a sodium citrate enema can be considered.

To help prevent recurrence of constipation, laxative treatment should be continued for several months. As defecation becomes more regular with treatment, the effect of laxatives on frequency or urgency of defecation gradually increases. The laxative dose can then be carefully reduced, usually without symptomatic relapse. One practical strategy is to advise parents to maintain the most effective dose of laxative until defecation becomes too frequent or too urgent and then to reduce the dose slowly over a few months.

\section{Bowel evacuation}

Bowel evacuation may be necessary if: a trial of laxatives fails; the colon is impacted; or the child also experiences pain, nausea or vomiting, in addition to constipation. Ideally, such treatments (which include bowel cleansing solutions taken orally, suppositories or enemas, or manual evacuation under anaesthesia) should only be attempted by a specialist.
Bowel cleansing solutions (normally used to clear the bowel before investigations or bowel surgery) are powders made into a solution with water and then taken by mouth; few are licensed for use in young children or for the treatment of constipation. Preparations include:

- sodium picosulfate/magnesium citrate

- polyethylene glycol

- magnesium citrate

- sodium dihydrogen phosphate dihydrate.

However, children may not easily tolerate these solutions, some of which require swallowing a large volume of fluid. The solutions are sometimes given via a nasogastric tube but insertion of the tube can be stressful for the child and is hazardous if inserted incorrectly. These preparations may cause distress, nausea, vomiting, colicky pain or urgent bowel movements, may cause fluid and electrolyte imbalance, especially in small children or in the presence of renal impairment.

If oral administration is ineffective or not tolerated, it may be worth trying rectal treatment. However, administration of suppositories and enemas can be difficult, not least because the child may find the treatments unpleasant, and has to remain still while the product is retained. If rectal preparations are required, small-volume sodium citrate enemas (micro-enemas) should be used in preference to the larger-volume phosphate enemas; some older children can be taught to self-administer micro-enemas. Children may be particularly anxious about rectal treatments if they have experienced anal pain or abuse and may interpret rectal administration as punishment, especially when the enema is presented as a threat. Sedation using midazolam or temazepan may allow enemas to be used without a lasting memory of distress but repeated use of such sedation may make parents worry about possible dependence.

Manual evacuation under a general anaesthetic may be the only option if all other treatments fail, if there is faecal impaction with signs of intestinal obstruction or pressure effects on the bladder leading to urinary retention.

\section{Biofeedback training}

Around $50 \%$ of children with chronic constipation show abnormal defecation dynamics. ${ }^{10}$ Biofeedback training aims to treat these problems, which can continue for several months despite laxative treatment. Such training teaches muscle relaxation using anorectal monitoring instruments to amplify physiological processes and to make physiological information accessible to the child's consciousness; it can only realistically be attempted in children old enough to understand the procedure. Randomised studies suggest that adding biofeedback training to conventional treatment (laxatives, counselling and toilet training), in children with chronic constipation and involuntary soiling, helps improve defecation dynamics ${ }^{10,11}$ but without a consistent increase in clinical recovery rates..$^{10,11,12}$

\section{When to refer}

Referral to a local paediatrician is probably advisable if:

- constipation is prolonged (over six months' duration)

- treatment in general practice has not been effective 
- there is frequent soiling and distress

- there is doubt as to the cause of the symptoms

- the condition is interfering with the child's schooling and social relationships.

Paediatricians working closely with the primary care team, including the community pharmacist, and the community paediatric nursing team can build a support network for the child and family. Paediatric nurses should have good communication with school nurses and can help teachers cope with children prone to faecal soiling. Health visitors and social workers can play a key role by visiting the family at home to observe domestic circumstances, such as mealtimes, toilet arrangements and general child-rearing. If there are other behavioural difficulties such as temper tantrums, sleep disorders or hyperactivity, referral for psychological help may be most effective, and this is best initiated by the paediatrician.

\section{Counselling and support}

Few children will voluntarily discuss their constipation or incontinence with even their closest friends and many parents keep these problems to themselves. It is crucial, therefore, that the child and family are offered counselling and support throughout management. Frequent problems that need addressing include:

- parental distress

- low self-esteem of the child due to embarrassment and teasing, especially at school

- poor adherence to toileting routines and medication regimens.

So while referral to a child psychologist or psychiatrist may be unnecessary, every child should have the psychosocial aspects of their problem considered. Psychological therapy is best started gently, with reassurance and simple counselling, and then increased according to need and the availability of resources. Everyone dealing with children who have defecation difficulties must be sensitive to the child's fears and aim to increase the child's involvement and motivation in the management of the problems. A wide range of incentive charts can be used to reward adherence to toileting routines and taking medication, as well as for successful defecation. However, these measures will be fruitless if the child is too young to appreciate them or too old to be impressed by them. Also, if colonic impaction is not recognised early, the continuing lack of treatment success may deter the child from co-operating further. A booklet, Childhood soiling - a guide for parents, has been designed for the British charity ERIC*.

\section{Conclusion}

In healthy children, there is a wide variation in the frequency of defecation and the hardness of stools. Key indications for the treatment of constipation are pain on defecation, severe straining or overflow incontinence and soiling. With mild symptoms, simple parental reassurance or dietary advice may

* Enuresis Resource and Information Centre www.enuresis.org.uk be all that is required and this should be offered by the primary care team. A child presenting with more severe symptoms and/ or psychosocial problems may well need further treatment, which often requires the involvement of a skilled team of doctors, nurses, social workers and psychologists, working together with the child, their parents and teachers. Not all of the drugs commonly used to treat constipation are licensed for use in young children nor have they been investigated in appropriate trials. However, if dietary treatment fails, it is sensible to use laxatives, starting with stool softeners or bulk laxatives and then moving on to stimulant laxatives, using a combination of drugs where necessary. Laxatives may be needed for some months and doses should only be reduced slowly to prevent re-impaction. If laxatives fail, bowel evacuation techniques (such as oral bowel cleansing solutions, enemas or manual evacuation) may be needed but should only be used under the supervision of a paediatrician. Adding biofeedback training to the use of laxatives, counselling and toilet training does not seem to increase the chances of longterm recovery from chronic constipation and soiling.

Adapted with permission from an original article in Drug and Therapeutics Bulletin 2000;38:57-60. (Published by Consumers' Association, 2 Marylebone Road, London, NW1 4DF, United Kingdom.)

\section{ACKNOWLEDGEMENT}

The Editorial Executive Committee of Australian Prescriber thanks Dr Tony Catto-Smith, Director, Department of Gastroenterology \& Clinical Nutrition, Royal Children's Hospital, Melbourne, for his helpful comments on this article.

\section{REFERENCES}

[R = randomised controlled trial $]$

1. Baker SS, Liptak GS, Colletti RB, et al. Constipation in infants and children: evaluation and treatment. A medical position statement of the North American Society for Pediatric Gastroenterology and Nutrition. J Pediatr Gastroenterol Nutr 1999;29:612-26.

2. Weaver LT, Steiner H. The bowel habit of young children. Arch Dis Child 1984;59:649-52.

3. Iacano G, Cavataio F, Montalto G, et al. Intolerance of cow's milk and chronic constipation in children. N Engl J Med 1998;339:1100-4.

4. Clayden GS, Lawson JON. Investigation and management of longstanding chronic constipation in childhood. Arch Dis Child 1976;51:918-23.

5. Loening-Baucke VA. Sensitivity of the sigmoid colon and rectum in children treated for chronic constipation. J Pediatr Gastroenterol Nutr 1984;3:454-9.

6. Royal College of Radiologists. Making the best use of a department of clinical radiology. Fourth edition. London: The Royal College of Radiologists; 1998.

7. Papadopoulou A, Clayden GS, Booth IW. The clinical value of solid marker transit studies in childhood constipation and soiling. Eur J Pediatr 1994;153:560-4.

8. Clayden GS. Childhood constipation. Paediatric practice guidelines. London: British Paediatric Association; 1994.

9. Perkin JM. Constipation in childhood: a controlled comparison between lactulose and standardized senna. Curr Med Res Opin 1977;4:540-3. (R)

10. Loening-Baucke V. Modulation of abnormal defecation dynamics by biofeedback treatment in chronically constipated children with encopresis. J Pediatr 1990;116:214-22. (R)

11. van der Plas RN, Benninga MA, Büller HA, et al. Biofeedback training in treatment of childhood constipation: a randomised controlled study. Lancet 1996;348:776-80. (R)

12. Loening-Baucke V. Biofeedback treatment for chronic constipation and encopresis in childhood: long-term outcome. Pediatrics 1995;96: 105-10. (R) 